\title{
Prevalencia de burnout en auditores gubernamentales de México, perspectiva de género
}

\author{
The prevalence of occupational burnout in the government auditors of Mexico, \\ a gender perspective
}

\author{
Juan Carlos Moreno Jiménez, Mara Maricela Trujillo Flores† \\ Fernando Lámbarry Vilchis* \\ Instituto Politécnico Nacional, México
}

Recibido el 4 de abril de 2017; aceptado el 28 de agosto de 2017

\section{Resumen}

Los auditores gubernamentales en México enfrentan agotadoras cargas de trabajo que los predispone a ocasionarles problemas psicosociales como es el Síndrome de Quemarse por el Trabajo (SQT-Burnout), entre otros. No obstante para esta profesión, el burnout no ha sido estudiado con profundidad como lo ha sido para algunas otras (especialmente aquellas que pertenecen al sector educativo y de la salud) y aún menos desde la perspectiva diferenciada por la variable género. El estudio cuantitativo con carácter no generalizable implicó aplicar el Maslach Burnout Inventory-General Survey (MBI-GS) a 557 auditores del ámbito federal y subnacional, la literatura especializada ha demostrado que no se trata únicamente de un fenómeno contingente. Para contrastar la hipótesis planteada Ho: las distribuciones de burnout entre hombres y mujeres son iguales, fue a través del método U de Mann-Whitney. Se concluye que en los auditores gubernamentales es mayor el burnout en las mujeres que en los hombres.

Código JEL: J29, M49

Palabras clave: Burnout; Género; Auditores; Inventario de burnout de maslach

\footnotetext{
*Autor para correspondencia Correo electrónico: flambarry@ipn.mx (F. Lámbarry Vilchis) La revisión por pares es responsabilidad de la Universidad Nacional Autónoma de México. http://dx.doi.org/10.22201/fca.24488410e.2018.1475 0186- 1042/C 2019 Universidad Nacional Autónoma de México, Facultad de Contaduría y Administración. Este es un artículo Open Access bajo la licencia CC BY-NC-SA (https://creativecommons.org/licenses/by-nc-sa/4.0/)
} 


\begin{abstract}
Government auditors in Mexico have tiring workloads that, beyond the physical and emotional exhaustion, predispose them to psychosocial problems such as Occupational Burnout, among others. Nevertheless, burnout has not been thoroughly studied for this profession as it has been done with others (especially those that pertain to the education and health sectors), and even less so from the approach differentiated by the gender variable. The quantitative study of a non-generalizable nature entailed the implementation of the Maslach Burnout Inventory-General Survey (MBI-GS) to 557 auditors in the federal and sub-national areas. It is an instrument based on the tridimensional model by Maslach and Jackson, which considers emotional exhaustion, feelings of cynicism and detachment from work, and the sensation of professional inefficiency and a lack of achievement. Specialized literature in this matter has demonstrated that it is not just a contingent phenomenon. Through the U Mann-Whitney method, the hypothesis Ho that was presented stating that the distributions of burnout between men and women are equal is contrasted. It is concluded that for government auditors, the burnout syndrome is more prevalent in women than in men.
\end{abstract}

JEL code: $\mathrm{J} 29, \mathrm{M} 49$

Keywords: Burnout; Gender; Auditors; Maslach burnout inventory

\title{
Introducción
}

La globalización ha traído una nueva situación social hacia la orientación del trabajador dentro del contexto laboral, De la Garza (2013) esboza problemas como son la calidad del empleo, la ocupación, los salarios, las políticas estructurales, la actividad de los actores que intervienen en los procesos y la forma en que se dan éstos en el escenario laboral, traduciéndose en un deterioro de las condiciones de vida y de trabajo, ocasionando mayores conflictos en los trabajadores. Aún bajo las condiciones adversas del escenario laboral descrito, los trabajadores que poseen empleo asalariado y de baja remuneración tratan de consérvalo a toda costa, ante lo anterior se ha sugerido una expansión de conceptos para el estudio del trabajo, producción, control y de las relaciones laborales (De la Garza y Campillo, 1998). En la actualidad debe interesar el trabajo que se da en la convergencia con el cliente, con el usuario, es decir, quien tiene que ver con los servicios, con el "cara a cara" entre el cliente y el productor (Sánchez, 2012).

En este contexto, las condiciones laborales del entorno actual han propiciado el surgimiento de problemas y factores psicosociales con repercusiones para los individuos y para las organizaciones; dichas características del mundo laboral contribuyen a incrementar el estrés de los trabajadores en el siglo XXI (Schwartzmann, 2004) a lo que Shirom (2009, p. 52) afirma que los trabajadores "experimentan una mayor inse- 
guridad laboral, exigencia de excesivas horas de trabajo, la necesidad de un continuo reentrenamiento (...) cambio en las tecnologías de la información y la poca claridad de la línea que separa el trabajo de la vida hogareña”, aspectos que para el caso mexicano se replican en el escenario laboral y de vida personal, ocasionándoles fenómenos como es el burnout, entre otros problemas y riesgos psicosociales.

Burnout es una combinación de dos palabras inglesas, burn que significa quemadura y out que significa fuera, esto da la idea de quemarse profesionalmente hasta el agotamiento, en la literatura podemos encontrar que existen al menos 17 denominaciones en español para describir el fenómeno (Gil-Monte, 2003) recomendándose el empleo del término "síndrome de quemarse por el trabajo" no obstante la falta de consenso para su uso, se sigue dando preeminencia al término en lengua inglesa (burnout). En la literatura especializada se puede encontrar el burnout con diferente terminología, como síndrome de estar quemado (una aproximación a su traducción del término anglosajón), síndrome de quemazón, desgaste profesional y desgaste psíquico, que son términos que vienen a ser equivalentes. Es Balseiro (2010) quien argumenta que el síndrome de burnout es un tipo específico de estrés laboral padecido por aquellos que en su voluntad por adaptarse y responder a un exceso de demandas y presiones laborales se esfuerzan de un modo intenso y sostenido en el tiempo con una exigencia y tensión que originan importantes riesgos de contraer enfermedad y afectar negativamente el rendimiento y la calidad en el servicio. De esta forma, el síndrome presupone la pérdida de la ilusión por el trabajo (Gil-Monte, 2002a; Moriana y Herruzo, 2004; Ordenes, 2004; Aranda, 2006). Se presenta como secuela del estrés laboral crónico con síntomas que van desde el agotamiento físico, mental y emocional hasta relaciones conflictivas interpersonales (Preciado, Salas, Franco y Vázquez, 2010). En el ámbito organizacional, el fenómeno radica en una alta movilidad laboral y disminución del rendimiento, ocasionando grandes costos socioeconómicos para la organización (Shirom, 2003; Volpp y Grande, 2003).

La presencia de burnout se ha confirmado en áreas de servicio cuya actividad implica que los trabajadores establezcan contacto con clientes, usuarios o alguna interrelación con otras personas (Peinado y Garcés, 1998; Gil-Monte, 2002a; Moriana y Herruzo, 2004; Ordenes, 2004; Aranda, 2006; Gil-Monte, 2006). En el sector público, una de estas áreas que da indicios sobre la existencia del burnout es el campo de la auditoría gubernamental, en gran parte por sus características laborales de extenuantes cargas de trabajo (Zaldúa, Lodiue y Koloditzky, 2000; Campos, Jiménez, Martín y Martínez, 2007). Asimismo, Gil-Monte y Peiró (1997) y Gil-Monte (2003), a partir de sus investigaciones ratifican que la ambigüedad de rol (asociado a los resultados esperados) es un predictor de la realización personal en el trabajo, el conflicto de rol (realización de tareas incompatibles) conlleva al cansancio emocional, de igual forma la sobrecarga de rol 
(demandas sin tiempo suficiente para realizarlas) está asociada al síndrome de burnout. Lo anterior también es apoyado en autores como Burke y Greenglass (1995), Shirom (2009). Igualmente, Guillén y Santamaría (2012) asocian al burnout con la cantidad de horas de trabajo, encontrando correlación con el cansancio emocional en el trabajador. Del mismo modo, Sefert, Jayaratne y Chess (1991) plantean que la falta de recompensas tanto económicas como sociales son predictores de despersonalización. Lo anterior se asocia a la figura del auditor, el cual es un profesionista con una alta cualificación que está sometido por lo delicado de su quehacer a un alto grado de responsabilidad, la cual tiene que desempeñar en lapsos de tiempo muy cortos, tomando en cuenta que en ciertos casos se tienen que auditar temáticas complejas, muestras representativas y una enorme cantidad de documentos en la búsqueda de resultados específicos que no siempre se obtienen. La evidencia documental reporta que el fenómeno es mayor en las mujeres (Maslach, Jackson y Leiter, 1996; Cámara y Cuesta 2005; Boydak, 2009), aunque la relación entre género y burnout no es siempre clara, es ambigua y con resultados inconsistentes (Guerrero y Rubio, 2005; Caro-Hernández, 2006; Purvanova y Muros, 2010). El método de investigación empleado en este estudio se fundamentó en el modelo de Maslach Burnout Inventory-General Survey MBI-GS, el más empleado en la literatura internacional (Caro-Hernández, 2006; Oramas, González y Vergara, 2007). La hipótesis de investigación planteada consistió en contrastar si las distribuciones del burnout entre hombres y mujeres son iguales, mediante el estadístico U de Mann-Whitney.

\section{Modelos cuantitativos del burnout: el Maslach Burnout Inventory}

Desde la etiología se valora al burnout como un proceso multicausal y altamente complejo ocasionado por ambientes laborales donde prevalece sobrecarga de trabajo, carencia de incentivos y mecanismos de promoción, excesiva burocratización, cambios de turnos y horarios laborales frecuentes, rotación constante en los puestos y cargos, salarios injustos y constantes estrategias de reducción de costos y de personal (Zaldúa et al., 2000; Martín et al., 2007). Desde la perspectiva de la psicología se desasocia al burnout con la fatiga y se asocia con la desmotivación emocional y cognitiva del sujeto provocado por el abandono de intereses que en un determinado momento le fueron importantes (Moreno y Peñacoba, 1999) es su percepción de discrepancia entre los esfuerzos que realiza y lo que consigue (Farber, 1983) lo cual le provoca alteraciones psicofisiológicas, además de repercutir negativamente en las organizaciones donde laboran (Gil-Monte y Peiró, 1997; Gil-Monte, 2005; Gil-Monte, Núñez-Román y Selva-Santoyo, 2006).

Desde una perspectiva psicosocial se considera al burnout como un proceso consecuencia del estrés laboral crónico refiriendo diferencias en cuanto a como se establece a nivel sintomático y su posible intervención, bajo estas premisas se fundamentan los 
modelos de Golembiewski, Munzenrider y Carter (1983), Price y Murphy (1984), Burke (1987), Leiter y Maslach (1988), Lee y Ashforth (1993) principalmente. Existen también modelos compresivos que refieren variables orientadas a comprender la etiología del síndrome por medio de antecedentes y consecuentes del fenómeno, ejemplo de ello son los estudio de Cox, Kuk y Leiter (1993), Hobfoll y Freedy (1993), Winnubst (1993), Tomás, Lima y Pacheco (2015).

De acuerdo con Quiceno y Vinaccia (2007) los modelos de desarrollo del síndrome de quemarse por el trabajo pueden dividirse en modelos comprensivos, es decir, elaborados desde la teoría sociocognitiva del yo, desde la teoría del intercambio social y desde la teoría organizacional y los modelos de proceso (que considera al modelo tridimensional de MBI-HSS, el modelo de Edelwich-Brodsky, el modelo de Price-Murphy y el modelo de Gil-Monte), este último ha ido adquiriendo una importante notoriedad (en España inicialmente y después a nivel internacional) a la par su instrumento el Cuestionario para la Evaluación del Síndrome de Quemarse por el Trabajo (CESQT) que propone 20 ítems agrupados en 4 dimensiones (desgaste psíquico, indolencia, culpa e ilusión), otros instrumentos que están intentando plantear nuevas perspectivas de evaluación que superen las limitaciones conceptuales y psicométricas del MBI son el Copenhagen Burnout Inventory-CBI (Kristensen, Borritz, Villadsen y Christensen, 2005) y el Oldenburg Burnout Inventory-OLBI (Halbesleben y Demerouti, 2005).

No obstante todos los señalamientos, el modelo tridimensional de Maslach y Jackson y su instrumento el Maslach Burnout Inventory (MBI) es el más utilizado para cuantificar al burnout, (Oramas et al., 2007) mismo que ha sido legitimado al español por Gil-Monte (2002b) y validado en España por Gil-Monte y Peiró (1999) mientras que en Chile por Buzzetti-Bravo (2005). Este modelo cuenta con tres versiones oficiales consecuencia de una reelaboración teórica del constructo por la ambigüedad de sus factores que resulta al aplicarlo a diferentes profesiones (Leiter, Clark y Durup, 1994; Auné, Abal y Attoresi, 2015; Salessi y Omar, 2016) 1. El MBI Human Services Survey (HSS) de 1981, enfocado a áreas de servicios humanos que cuenta con 22 reactivos, 2. El MBI Educators Survey (ES) orientado al sector educación de 22 reactivos (Maslach y Schaufeli, 1993), finalmente 3. El MBI General Survey (GS) de 1996 empleado en profesiones no asistenciales y cuenta con 16 reactivos (Maslach, Jackson y Leiter, 1996) en el cual se redefine al burnout como una crisis en relación con el propio trabajo (Maslach et al., 1996; Oramas et al., 2007) que se cuantifica a través de tres escalas:

-Desgaste emocional: se valora la sensación de agotamiento, de estar acabado al final de la jornada, cansancio, fatiga, tensión de origen emocional, que se manifiesta en forma de falta de energía para realizar el trabajo.

- Cinismo: refleja la actitud de indiferencia, devaluación y distanciamiento ante el propio trabajo y el valor y significación que se le pueda conceder. Se manifiesta en 
forma de pérdida del interés laboral, del entusiasmo y cuestionamiento de la valía de la labor que se realiza.

- Eficacia profesional: relacionada con las expectativas que se tienen y la profesión que se desempeña, se expresa en las creencias del sujeto sobre su capacidad de trabajo, su contribución eficaz en la organización laboral, de haber realizado cosas que realmente valen la pena y de realización profesional.

Si bien es cierto que MBI es el instrumento que más aceptación ha tenido en el campo de medición del síndrome de quemarse por el trabajo respecto a las características psicométricas se han señalado los problemas derivados de utilizar criterios poblacionales como puntos de corte del síndrome o la baja consistencia interna de la dimensión cinismo en muestras no anglosajonas, además autores como Olivares y Gil (2009) han mostrado diversas debilidades en el instrumento, lo que cuestiona la viabilidad de su aplicación en diferentes contextos (Juárez-García, Idrovo, Camacho-Ávila y Placencia-Reyes, 2014). Por su parte Moreno, Trujillo, Rivas y Lámbarry (2014) en su investigación realizan un estudio sobre la identidad, uso y aplicación del MBI en México, principalmente en Instituciones de Educación Superior, reafirmando la amplia aceptación y popularidad del instrumento.

\section{El burnout y su divergencia en el género: la investigación en el sector público en México}

Desde 1985, Maslach y Jackson (1985) señalaron que el estudio de las diferencias de burnout en hombres y mujeres son muy pequeñas o no existen y que en consecuencia el género es un factor que explica una parte pequeña en su prevalencia y varianza, pese a ello, las autoras evidenciaron una intensidad ligeramente mayor de agotamiento emocional en las mujeres y una mayor intensidad de baja realización profesional en los hombres, su explicación se atribuye al condicionamiento de género (Oramas et al., 2007). Al respecto Reygadas (2004) establece que persisten las asimetrías e inequidades en las relaciones de género actualmente en múltiples escenarios, pese a lo anterior, el autor expresa que la mujer ha adquirido una mayor participación en todas las esferas de la vida económica, política y social, pero hasta la fecha las principales posiciones de poder y privilegio siguen siendo ocupadas por hombres. A medida que se asciende en la escala de importancia de esas posiciones se reduce la participación femenina, lo anterior ilustrado en la estructura política, observándose en instancias como el mercado, los gobiernos y las empresas que están caracterizadas por la explotación, la corrupción y las relaciones asimétricas (Arnold y Spedding, 2005).

Desde la perspectiva de distintos grupos ocupacionales, se observa por ejemplo que en el sector educativo, el sexo femenino es el que presenta una mayor prevalencia del síndrome (Martínez y Salanova, 2003; Salanova, Llorens y García-Renedo, 2003) mientras 
que, en oficiales de policía (hombres y mujeres) no se reportan diferencias significativas en los niveles de burnout (McCarty, Solomon-Zhao y Garland, 2007). En una muestra de trabajadores inmigrantes en España poco cualificados (que carecen o presentan una baja formación especializada para desempeñar una determinada actividad o trabajo), se encontró que no existe diferencia entre hombres y mujeres y su nivel de burnout (Moreno-Jiménez, Ríos-Rodríguez, Canto-Ortíz, San Martín-García y Perles-Nova, 2010) resultado interesante, si se toma en cuenta que su incursión en el mercado laboral se da bajo condiciones precarias, en empleos temporales y con bajos salarios.

Para Purvanova y Muros (2010) la literatura reportada para conocer la divergencia en burnout entre mujeres-hombres, arroja resultados inconsistentes, para dar mayor claridad realizaron un meta-análisis en 180 estudios, entre sus principales resultados encontraron que las mujeres presentaron mayores niveles en agotamiento emocional que los hombres, mientras que los hombres presentaron mayor nivel de despersonalización que las mujeres, aunque las diferencias son mínimas, otro resultado revelador es que las diferencias de género son mayores en los Estados Unidos con relación a los reportados en la Unión Europea. Es por ello que la relación entre género y burnout no es siempre clara, es ambigua y con resultados inconsistentes que dependen del sector y contexto en estudio. Ante la pregunta de ¿quiénes se encuentran más quemados por su trabajo, los hombres o las mujeres? Malach-Pines (2005) responde que básicamente se encuentra en función de la cultura, la profesión, el trabajo, otras tensiones encontradas y la medida utilizada para evaluar el nivel de burnout.

En el caso mexicano, una revisión sistemática sobre burnout realizada por Juárez-García, et al., (2014) afirman que el instrumento más utilizado en México es el Maslach Burnout Inventory, identificándolo en el $90 \%$ de los 64 estudios analizados, pero sólo en 10 de ellos encontraron que las variables demográficas asociadas al síndrome y más frecuentemente señaladas son el género y la antigüedad. Sobresalen en este contex to 4 estudios que hacen uso del MBI pero ninguno de ellos se orienta al campo de la auditoría gubernamental, ni analizan la relación entre burnout y género: uno de ellos se realiza a 875 agentes de tránsito por Aranda, Pando, Salazar, Torres y Aldrete (2009), quiénes concluyen que estos trabajadores cuentan con un sistema de apoyo inadecuado, causante en gran medida del burnout y con repercusiones graves eventualmente en su salud. Otro es efectuado a 29 directivos de la Comisión Federal de Electricidad por Cabrera, Ruiz, González, Vega y Valadez (2009), los cuales no reportan prevalencia del síndrome concluyendo que el personal con responsabilidades específicas tiende a frustrarse al percibir que dan más de lo que reciben, lo que se traduce en un agotamiento emocional.

Por su parte Valdez (2009) al estudiar a 1,391 trabajadores penitenciarios, reportan prevalencia del burnout del $4.10 \%$, y concluyen que es baja. Finalmente, Aranda, Rendon 
y Ramos (2011) estudian a 466 trabajadores de trenes eléctricos obteniendo una prevalencia de $39.40 \%$, por lo que concluyen que la sub-escala más incidente en el burnout es la falta de realización con el $28.3 \%$ del total, seguida por agotamiento emocional con $14.2 \%$, y despersonalización con $9.2 \%$, además de una significativa asociación entre el agotamiento emocional del trabajador con una percepción de sentirse enfermo.

\section{Organización del sector y condiciones laborales del auditor gubernamental en México}

Desde 1980, la importancia de la actividad realizada por los auditores se ha incrementado notablemente (Power, 1997), hoy en día es prácticamente improbable identificar un área o sector gubernamental que no se audite. En México, la sociedad tiene altas expectativas sobre el trabajo realizado por los auditores, al atribuirles un rol estratégico para combatir la corrupción, detectarla y/o eliminarla (Munro, 2004; Kahn, 2006) no obstante, esta idea se encuentra alejada de la realidad, pues más bien su labor consiste en investigar, consultar, revisar, verificar, recopilar y evaluar la información institucional para valorar el grado de cumplimiento en sus actividades y atribuciones y su apego al marco normativo (Arellano y Coronilla, 2012); por otro lado, el trabajo de los auditores se cuestiona cotidianamente al creer que son cooptados por la corrupción al no detectar ni denunciar los grandes escándalos financieros en un país (DeFond y Francis, 2005), sin olvidar que sus resultados son puestos en duda ante los señalamientos sobre posibles conflictos de interés (Moore, Tetlock, Tanlu y Bazerman, 2006) y que inherentemente su actividad puede presentar sesgos que entorpecen su labor y decisiones (Bazerman, Loewenstein y Moore, 2002) además, las reglas y normas internacionales de auditoría (Prather-Kinsey, 2006) que son aplicadas en México suponen una preparación continua y especializada para su uso. Por lo tanto es como se observa, una actividad con una presión social permanente sobre la que se imponen altas expectativas sobre su alcance y resultados. A este respecto, es necesario tener en cuenta que los cambios en torno al trabajo y las organizaciones (sobrecarga y prolongadas e intensas jornadas de trabajo) dañan la calidad de vida laboral tal como lo establece Bagtasos (2011).

México cuenta con una amplia tradición en materia de auditoría gubernamental, pues en todo el sector público existen órganos de auditoría, rendición de cuentas, control interno y fiscalización. En el poder ejecutivo, por ejemplo se tiene a la Secretaría de la Función Pública y a los más de 200 Órganos Internos de Control de las dependencias y entidades de la Administración Pública Federal. En el Poder Legislativo se encuentra la Auditoría Superior de la Federación, una contraloría interna en la Cámara de Diputados y una contraloría interna en el Senado. Para el caso particular del personal del ámbito federal que realiza actividades vinculadas con la auditoría Pardinas (2009) señala que en la Secretaría de la Función Pública existen 2400 trabajadores que sumados con los empleados de los Órganos Internos de Control se tienen cerca 
de 12000 dentro de la Administración Pública Federal realizando una labor conexa directa e indirecta con la auditoría.

Por su parte en la Auditoría Superior de la Federación laboran alrededor de 3 mil empleados de estructura y prestadores de servicios profesionales. En el Poder Judicial se tiene a la Contraloría de la Suprema Corte de Justicia de la Nación, la Contraloría del Poder Judicial de la Federación (presidida por el Consejo de la Judicatura Federal) la Contraloría Interna del Tribunal Electoral del Poder Judicial de la Federación, la Contraloría Interna de Tribunales Colegiados de Circuito, en los Órganos Autónomos se tienen contralores internos.

A nivel local, se encuentran las Secretarías de Contraloría (o su equivalente) en cada estado y las Entidades de Fiscalización Superior Locales; de acuerdo con el Centro Universitario de Ciencias Económico Administrativas de la Universidad de Guadalajara y el Instituto Mexicano de la Competitividad CUCEA-IMCO (citado en Figueroa y Palacios, 2015) existen 4268 auditores en las 32 entidades federativas con un promedio nacional de 133 auditores por Estado. En el ámbito municipal determinar el número de auditores o empleados relacionados con esta actividad es complejo, derivado de la gran diversidad que existe entre los municipios de México, basta señalar que existen en los ayuntamientos, síndicos y/o contralores que realizan funciones similares a las de un auditor (Ugalde, 2002).

\section{Materiales y método}

El estudio fue de corte transversal con carácter no generalizable e implicó aplicar el MBI-GS a los auditores gubernamentales seleccionados por solicitud expresa, vía correo electrónico con el texto siguiente:

El "Cuestionario de Síndrome de Quemarse por el Trabajo”, tiene como propósito conocer el nivel de Burnout que prevalece en su área de trabajo. Estimado auditor, a continuación se presentan algunas afirmaciones en relación a su actividad y sobre las consecuencias que se derivan de esa relación para usted como profesional y como persona. Piense con qué frecuencia le surgen a usted esas ideas o con qué frecuencia las siente, teniendo en cuenta la escala que se le presenta a continuación (...). Para responder rodee con un círculo la alternativa (el número) que más se ajusta a su situación.

Esta información es de carácter reservado, confidencial y sólo será utilizada para fines de investigación, por lo que la veracidad de sus respuestas es importante.

La muestra inicial consistió en aplicar el instrumento a 557 auditores de organizaciones del sector público en México (variables sociodemográficas tabla 1) del 27 de Julio al 2 de Agosto 
de 2016. Se realizó una muestreo basado en cuotas con auditores del ámbito subnacional (Entidades de Fiscalización Superior Local "EFSL" y de las Contralorías de los Estados "C" de las 32 entidades federativas) quedando integrada por 426 servidores públicos de los Estados y 131 auditores del ámbito federal (tablas A1 y A2, en anexos), de ellos 42 cuestionarios resultaron no válidos debido a que no se obtuvo respuesta, el error muestral fue de $3.86 \%$, nivel de confianza a intervalo: $95 \%, z=1.96 ; \mathrm{p}=\mathrm{q}=0.5$. De la aplicación de los cuestionarios semiestructurados resultaron 515 válidos, de los cuales 295 fueron respondidos por hombres $(57.3 \%)$ y 220 por mujeres $(47.7 \%)$, el $88.35 \%$ de los auditores cuenta con licenciatura como el nivel máximo de estudios, el $82.1 \%$ (423) tienen una edad entre los 18 a 45 años, aunque con un promedio de antigüedad cercano a los 4 años y trabajan aproximadamente 51 horas a la semana.

Tabla 1

Caracterización de la muestra

\begin{tabular}{|c|c|c|c|}
\hline \multicolumn{2}{|c|}{ Variables sociodemográficas } & \multirow{2}{*}{$\begin{array}{c}\text { Número } \\
220\end{array}$} & \multirow{2}{*}{$\begin{array}{c}\text { Porcentaje } \\
47.72\end{array}$} \\
\hline Ć́nor & Femenino & & \\
\hline Gen & Masculino & 295 & 57.28 \\
\hline \multirow{5}{*}{ Edad } & $18-25$ & 68 & 13.20 \\
\hline & $26-35$ & 231 & 44.85 \\
\hline & $36-45$ & 124 & 24.08 \\
\hline & $46-60$ & 75 & 14.56 \\
\hline & 61 o más & 17 & 3.31 \\
\hline \multirow{3}{*}{ Número de hijos } & Sin hijos & 299 & 58.06 \\
\hline & Con 1 hijo & 83 & 16.12 \\
\hline & Más de 1 hijo & 133 & 25.82 \\
\hline \multirow{4}{*}{ Estudios } & Elementales (Primaria, Secundaria) & 3 & 0.58 \\
\hline & Medios (Bachillerato, Preparatoria) & 9 & 1.75 \\
\hline & Licenciatura & 455 & 88.35 \\
\hline & Posgrado & 48 & 9.32 \\
\hline \multirow{3}{*}{ Antigüedad en su trabajo actual } & 1 año o menos & 174 & 33.79 \\
\hline & 2 a 4 años & 136 & 26.41 \\
\hline & Más de 4 años & 205 & 39.8 \\
\hline
\end{tabular}

Fuente: elaboración propia. 


\section{Escala de medición}

Se utilizó el cuestionario Maslach Burnout Inventory-General Survey (MBI-GS) adaptación al español de 16 ítems de (Moreno-Jiménez, et al., 2001) distribuidos en tres sub-escalas: desgaste emocional (5 items), cinismo (5 items) y eficacia profesional (6 ítems). En el juicio de expertos se validó la transcripción de los ítems a fin de que los auditores no presentaran dudas sobre los mismos y conservando la esencia de la escala original. Cada ítem fue valorado con una escala Likert de 7 dimensiones, en la que indican la frecuencia con la que han experimentado la situación descrita desde 0 (nunca) hasta 6 (todos los días), redactados como afirmaciones, a excepción de la dimensión eficacia profesional que fue valorada en forma inversa para cada ítem desde -6 (todos los días) hasta 0 (nunca). De aquí que el valor mínimo de la escala de medición del burnout es de -36, ya que el efecto real de la dimensión eficacia profesional resta a la prevalencia del burnout (es negativa), y el máximo es de $60^{1}$ (resultado de la suma de las dimensiones de agotamiento emocional y cinismo dado que la eficacia profesional presentara un valor de cero), ante ello, se consideraron tres niveles: bajo para puntuaciones de 1 a 7 , medio entre 8 y 15 y alto mayores a $16^{2}$. En cuanto a los valores de fiabilidad del MBI-GS el pretest inicial resultó en un coeficiente Alfa de Cronbach general de 0.901 (alta confiabilidad en la medición), mientras que para las sub-escalas coeficientes de 0.85 para eficacia profesional, 0.83 para agotamiento emocional y 0.74 para cinismo.

Con los datos obtenidos, se realizó un análisis factorial exploratorio de componentes principales con rotación Varimax. El coeficiente Kaiser-Meyer-Olkin obtenido fue de 0.903 y una prueba de esfericidad de Barlett con un Chi-cuadrado de 4 649.461; g.l. 120; $\mathrm{p}<0.000$. La finalidad fue identificar aquellos ítems que no estaban adheridos a su dimensión (Hair, Black, Babin, Anderson y Tatham, 1998; Lámbarry, Rivas y Trujillo, 2013) y el grado de dimensionalidad de sus respectivas cargas factoriales (tabla 2). Cada una de las sub-escalas presentaron un buen grado de unidimensionalidad, con cargas factoriales que superan valores por arriba de 0.4 (Landero y González, 2009). Derivado de esto, resultaron tres componentes principales que explican el $65.13 \%$ de la varianza del burnout, confirmándose la multidimensional (agotamiento emocional, cinismo y eficacia profesional) del constructo compuesto por 16 ítems.

\footnotetext{
${ }^{1}$ Cabe señalar, que el instrumento MBI-GS fue aplicado bajo la propuesta original de sus autores, por lo que la redacción de los ítems fueron considerados en forma de afirmación, no obstante, la dimensión eficacia profesional tal y como está considerada en el instrumento tiene un efecto negativo en el burnout.

${ }^{2}$ No existe una estandarización y se han señalado errores de medición alrededor de los puntos de corte con relación a la escala de medición de burnout, diversas investigaciones lo refieren (Olivares y Gil, 2009; Schaufeli, Leiter y Maslach, 2009; Fernández y Merino, 2014). Para este estudio se siguieron las indicadas.
} 


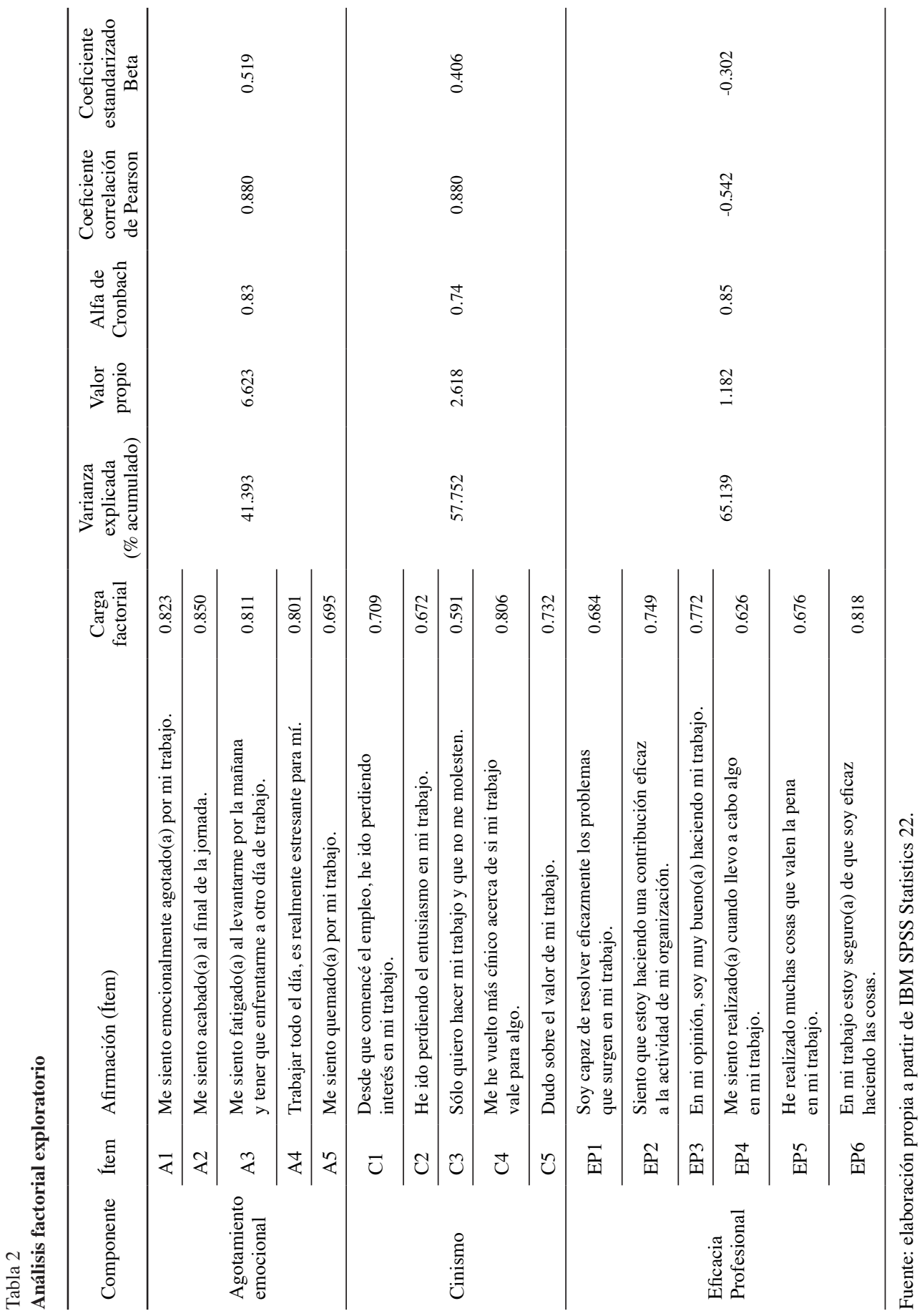


El agotamiento emocional y el cinismo se correlacionan positiva y significativa con el burnout, ambos con un valor de 0.880 , son también las dimensiones que inciden con mayor peso en la determinación del burnout al presentar coeficientes estandarizados de 0.519 y 0.406 respectivamente, además explican el $57.7 \%$ de la variabilidad del síndrome, caso contrario para la dimensión de eficacia profesional, que se correlaciona de forma negativa y significativa con el burnout a través de un coeficiente de -0.542 y un coeficiente estandarizado de -0.302 (tabla 2). En conjunto las tres dimensiones explican el 65.139 de la varianza del burnout.

En cuanto a los indicadores con mayor influencia en el burnout están: me siento acabado(a) al final de la jornada laboral (A2) con un coeficiente de 0.128; sólo quiero hacer mi trabajo y que no me molesten $(\mathrm{C} 3)$ con 0.126 y; trabajar todo el día, es realmente estresante para mí (A4) con 0.125 , mientras que el menos influyente es, dudo sobre el valor de mi trabajo (C5) con 0.088 . En este mismo sentido, el ítem me siento realizado(a) cuando llevo a cabo algo en mí trabajo (EP4) es el que más negativamente incide en el síndrome, con -0.077 seguido por, he realizado muchas cosas que valen la pena en mi trabajo (EP5) con -0.076.

\section{Resultados y discusión}

En total son $79(15 \%)$ auditores gubernamentales mexicanos los que padecen burnout; de ellos el 39\% son hombres y el 40\% mujeres, a pesar de que 436 (85\%) auditores aún no cuentan con algún indicio de padecerlo, se evidencia la existencia de burnout en los auditores mexicanos. De quiénes lo padecen, el $40.5 \%$ de ellos se encuentran en un nivel bajo, mientras que el $29.1 \%$ en un nivel medio y el $30.4 \%$ en niveles altos de burnout.

En consideración a la incidencia del burnout respecto del género, el $3.1 \%$ del total de los auditores que es el mayor porcentaje en la métrica del síndrome, tanto para hombres como para mujeres obtuvieron un nivel bajo, seguido por un $2.3 \%$ que padecen el síndrome en niveles altos, de igual forma para ambos sexos (tabla 3). No obstante, al ser la media -13.79 mayor en las mujeres que en los hombres, se infiere en primera instancia la incidencia en mayor grado en este género (tabla 4). 
Tabla 3

Tabulación cruzada: sexo vs nivel de burnout y nivel sub-escalas

\begin{tabular}{|c|c|c|c|c|c|c|c|c|c|c|c|}
\hline & \multicolumn{6}{|c|}{ Nivel B (\% de la tabla) Escala: -36 a 24} & \multicolumn{5}{|c|}{ Nivel AE (\% de la tabla) Sub-escala: 0 a 30} \\
\hline & \multicolumn{2}{|l|}{$\mathrm{Al}$} & $\mathrm{Ba}$ & $\mathrm{Me}$ & \multicolumn{2}{|r|}{ No } & \multicolumn{2}{|c|}{$\mathrm{Al}$} & $\mathrm{Ba}$ & \multirow{2}{*}{$\begin{array}{c}\mathrm{Me} \\
15-19\end{array}$} & \multirow{2}{*}{$\begin{array}{c}\text { No } \\
\text { Menor que } 10\end{array}$} \\
\hline Valores & Mayor qu & 16 & $1-7$ & $8-15$ & Mer & nor que 0 & Mayo & que 19 & $10-14$ & & \\
\hline Hombre & 2.3 & & 3.1 & 2.1 & & 49.7 & & & 7.8 & 8.0 & 30.3 \\
\hline Mujer & 2.3 & & 3.1 & 2.3 & & 35.0 & & & 9.1 & 7.4 & 16.5 \\
\hline \multicolumn{7}{|c|}{ Nivel C (\% de la tabla) Sub-escala: 0 a 30} & \multicolumn{5}{|c|}{ Nivel EP (\% de la tabla) Sub-escala: 0 a -36} \\
\hline Al & $\mathrm{Ba}$ & $\mathrm{Me}$ & & No & & & 1 & $\mathrm{Ba}$ & & & No \\
\hline Mayor que 19 & $10-14$ & $15-19$ & & Menor qu & 10 & Mayor & que -12 & -18 a- 23 & & -17 & Menor que -23 \\
\hline 2.3 & 4.9 & 3.1 & & 47.0 & & & 6 & 1.4 & & & 55.0 \\
\hline 2.3 & 4.9 & 3.5 & & 32.0 & & & 4 & 1.0 & & & 40.6 \\
\hline
\end{tabular}

Nota: B: burnout. AE: Agotamiento emocional. C: cinismo. EP: eficacia profesional; recordar que los valores de esta escala por el diseño del instrumento MBI-GS restan a la prevalencia del burnout (es negativa). $\mathrm{Al}$ : alto. Ba: bajo. Me: medio. No: No existe. Fuente: elaboración propia.

Tabla 4

Estadísticas: burnout, sub-escalas e indicadores

\begin{tabular}{|c|c|c|c|c|c|c|c|c|}
\hline \multirow{2}{*}{$\begin{array}{l}\text { Escalas (sub) } \\
\text { Género }\end{array}$} & \multicolumn{2}{|c|}{ Burnout } & \multicolumn{2}{|c|}{$\begin{array}{c}\text { Sub-escala: Agotamiento } \\
\text { emocional }\end{array}$} & \multicolumn{2}{|c|}{$\begin{array}{l}\text { Sub-escala: } \\
\text { Cinismo }\end{array}$} & \multicolumn{2}{|c|}{$\begin{array}{c}\text { Sub-escala: Eficacia } \\
\text { profesional }\end{array}$} \\
\hline & Hombre & Mujer & Hombre & Mujer & Hombre & Mujer & Hombre & Mujer \\
\hline Media & -17.93 & -13.79 & 10.50 & 12.75 & 4.88 & 6.06 & -33.31 & -32.60 \\
\hline $\begin{array}{l}\text { Desviación } \\
\text { Estándar }\end{array}$ & 15.34 & 15.66 & 8.06 & 7.96 & 6.16 & 6.52 & -4.60 & -4.82 \\
\hline Varianza & 235.341 & 245.152 & 65.026 & 63 & 38 & 43 & 21 & 23 \\
\hline Mínimo & -36 & -36 & 0 & 0 & 0 & 0 & -1 & -6 \\
\hline Máximo & 59 & 32 & 30 & 30 & 30 & 27 & -36 & -36 \\
\hline $\begin{array}{l}\text { Correlación de } \\
\text { Pearson }\end{array}$ & \multicolumn{2}{|c|}{$\mathrm{Na}^{*}$} & 0.870 & 0.888 & 0.871 & 0.889 & -0.642 & -0.579 \\
\hline $\begin{array}{l}\text { Coeficientes } \\
\text { estandarizados }\end{array}$ & \multicolumn{2}{|c|}{$\mathrm{Na}^{*}$} & 0.526 & 0.508 & 0.402 & 0.416 & -0.300 & -0.308 \\
\hline
\end{tabular}




\begin{tabular}{lcccccccc}
\hline Indicadores: & \multicolumn{2}{c}{ Burnout } & $\begin{array}{c}\text { Sub-escala: Agotamiento } \\
\text { emocional }\end{array}$ & \multicolumn{2}{c}{$\begin{array}{c}\text { Sub-escala: } \\
\text { Cinismo }\end{array}$} & \multicolumn{2}{c}{$\begin{array}{c}\text { Sub-escala: Eficacia } \\
\text { profesional }\end{array}$} \\
\hline Género & Hombre & Mujer & Hombre & Mujer & Hombre & Mujer & Hombre & Mujer \\
\hline Coeficientes & A5: & A2: & A2: & & C3: & C3: & EP4: & EP1: \\
estandarizados & $0.794 * *$ & $0.122 * *$ & $0.250^{* *}$ & A2: $0.241 * *$ & $0.329 *$ & $0.289 * *$ & $0.271 * *$ & $0.249 * *$ \\
beta & EP5: & EP4: & A5: & A5: $0.224 * * *$ & C1: & C4: & EP1: & EP6: \\
& $-0.562 * * *$ & $-0.073^{* * *}$ & $0.212 * * *$ & & $0.219 * * *$ & $0.211 * * *$ & $0.200 * * *$ & $0.190 * * *$ \\
\hline
\end{tabular}

Nota: Na* No aplica

** Coeficiente con mayor incidencia.

*** Coeficiente con menor incidenca.

Fuente: elaboración propia.

En cuanto a los coeficientes estandarizados Beta obtenidos de la regresión lineal múltiple, las sub-escalas inciden en el síndrome en forma muy similar con valores muy aproximados, independientemente del género del auditor; en mayor grado se encuentra agotamiento emocional y en menor grado eficacia profesional, tendencia que se visualiza también al observar el porcentaje de varianza explicada por cada sub-escala (tabla 2). A nivel indicador, A2, me siento acabado(a) al final de la jornada es el que mayor incide con un coeficiente estandarizado de 0.122 para las mujeres, mientras que, para los hombres resulta ser C3 sólo quiero hacer mi trabajo y que no me molesten con un valor estandarizado de 0.132 , aunque estos dos indicadores son los dos más determinantes del burnout para ambos sexos.

En la sub-escala agotamiento emocional, ambos géneros concentran el mayor porcentaje en un nivel alto; los hombres tienen el $11.3 \%$ y las mujeres el $9.7 \%$. Pese a ello, es el género femenino quien cuenta con el valor medio con 12.75 que supera al de los hombres de 10.5. En ambos, el indicador A2, me siento acabado(a) al final de la jornada, determinan aproximadamente el $25 \%$ de su agotamiento emocional. Mientras que en la sub-escala cinismo, es el mismo porcentaje de $4.9 \%$ para los hombres y las mujeres, lo que domina en un nivel medio, toda vez que la media del género femenino de 6.06 es mayor respecto del masculino de 4.88. El indicador C3, sólo quiero hacer mi trabajo y que no me molesten, para ambos géneros, determinan cerca del $30 \%$ de sus actitudes de cinismo. Finalmente, en el nivel alto de la sub-escala eficacia profesional es donde radican los mayores porcentajes de ambos géneros, es el masculino con el $55 \%$ el que supera notablemente al femenino con el $40.6 \%$, no obstante el valor medio en los hombres de 33.31 es ligeramente mayor que en las mujeres de 32.6. El indicador EP4, me siento realizado(a) cuando llevo a cabo algo en mi trabajo, es el que más determina la eficacia profesional para hombres, mientras que es EP1, soy capaz de resolver eficazmente los problemas que surgen en mi trabajo, para las mujeres; ambos indicadores determinan cerca del $25 \%$ de la eficacia profesional. 
En cuanto a la prueba de normalidad la distribución presentó un coeficiente de Kolmogorov Smirnov de 0.119 significativo a $0.01(0.000)$ para los hombres, mientras que de 0.096 significativo a $0.01(0.000)$ para las mujeres, por lo que se acepta la hipótesis alterna de que el burnout en la población difiere de una distribución normal tanto para los hombres como para las mujeres. De aquí que, para contrastar la hipótesis Ho: las distribuciones del burnout entre hombres y mujeres son iguales, se seleccionó la prueba U de Mann-Whitney, cuyo resultado fue significativo a 0.01 (0.001). Por lo que se acepta la hipótesis Ha: las distribuciones del burnout entre hombres y mujeres difieren. De tal forma se concluye que existe diferencia entre el burnout y la variable género. Toda vez que, el rango promedio para los 295 hombres es de 239.3, mientras que para las 220 mujeres es de 283.08 lo que confirma que el burnout tiende a ser mayor en las mujeres que en los hombres. No obstante, en cuanto al nivel de burnout que prevalece en los auditores mujeres y hombres es bajo (1-7), es la tendencia que se observa en las dimensiones de agotamiento emocional y de cinismo a diferencia de eficacia profesional, que es donde se presentan niveles altos del burnout pero que se correlaciona negativamente.

\section{Conclusiones}

Una primera impresión de este estudio es sobre el MBI y particularmente sobre la dimensión de eficacia profesional es su desconcertante interpretación negativa al valorarla conjuntamente con las otras dos dimensiones: cinismo y agotamiento emocional, quiénes inciden positivamente en la métrica del burnout, por lo que la eficacia profesional debe plantearse con una sintaxis de sus ítems en forma negativa y con ello evitar un análisis fragmentado de cada dimensión o bien evitar la interpretación de una escala negativa de valoración del burnout.

En relación a otros estudios se tiene que en Colombia con una muestra de 872 contadores públicos de 86 ciudades, se encontró que $54.8 \%$ presentan un nivel alto de burnout (Loaiza y Peña, 2013) en Estados Unidos y con 1681 auditores, contadores y consultores en temas fiscales se encontró que experimentan agotamiento emocional en niveles similares, pero son las mujeres las que reportan niveles más bajos de eficacia profesional y son los hombres los que presentan mayores niveles de despersonalización/ cinismo (Jones III, Guthrie y Iyer, 2012; Guthrie y Jones III, 2012) al comparar a los contadores con otros colectivos profesionales Fogarty, Singh, Rhoads y Moore (2000) encontraron que los valores medios reportados en agotamiento emocional son mayores a los experimentados por maestros y enfermeras, para la despersonalización los valores son similares a los residentes médicos; la eficacia profesional es más baja en relación 
a los abogados. Las mujeres auditoras mexicanas son el género que sensiblemente ostenta los mayores niveles del síndrome, lo que se observa con una pequeña diferencia al comparar el valor medio que supera escasamente al de los hombres, esto refuerza los resultados de Maslach, Jackson y Leiter (1996), Cámara y Cuesta (2005) y Boydak (2009) en sus estudios. En cuanto a las dimensiones agotamiento emocional y cinismo, son las mujeres auditoras quienes presentan los valores medios mayores respecto de los hombres, aunque ambos presentan niveles bajos de burnout, no obstante, esto indica que las auditoras perciben un mayor agotamiento emocional y cinismo que los hombres coincidiendo con la explicación de condicionamiento de género de Oramas et al., (2007).

Es la dimensión eficacia profesional donde el valor promedio del burnout en los auditores hombres supera marginalmente al de las mujeres, lo que se traduce que este género percibe una mayor realización profesional, reforzando los resultados de Purvanova y Muros (2010). En términos generales, es la dimensión que mayor valor medio presenta respecto de las otras dos y por su alto coeficiente correlacional negativo actúa como atenuante del burnout contrarrestando el efecto de las otras dos que tienen coeficientes correlacionales menores positivos, al grado de mantener niveles bajos de burnout en los trabajadores.

Derivado de lo anterior una primera conclusión que se delinea de este estudio es la prevalencia del burnout en los auditores mexicanos atribuible a tres componentes principales que explican el $65.13 \%$ de la varianza del burnout, confirmándose la multidimensional (agotamiento emocional, cinismo y eficacia profesional) del constructo.

Una segunda conclusión de este estudio es que hay diferencia entre el burnout y la variable género y se confirma que tiende a ser mayor en las mujeres que en los hombres.

Un tema pendiente a estudiar en este colectivo de auditores gubernamentales es la incidencia de variables cronológicas y sociodemográficas en el burnout, tales como, la carga de trabajo asignada que suele ser extenuante con un promedio de 51 horas semanales (aunque algunos auditores reportaron hasta 70 horas de trabajo y los tiempos cortos con relación al número de documentos a revisar) su antigüedad laboral que en este caso fue de 4 años derivado de una alta tasa de rotación y de su edad y el estatus civil. Además de una mayor investigación en grupos ocupacionales propensos a ser afectados por diversos riesgos psicosociales, sobre todo en el sector público mexicano, además de considerar otros instrumentos de medición como son el Cuestionario para la Evaluación del Síndrome de Quemarse por el Trabajo (CESQT) de Gil-Monte o la Escala Mexicana de Desgaste Ocupacional (EMEDO) de Uribe-Prado y con ello contrastar resultados considerando la variable género. 


\section{Referencias}

Aranda Beltrán, C. (2006). Diferencias por sexo, Síndrome de burnout y manifestaciones clínicas, en los médicos familiares de dos instituciones de salud. En Revista costarricense de salud pública, 15(29), 1-7.

Aranda Beltrán, C., Pando Moreno, M., Salazar Estrada, J., Torres López, T.M. \& Aldrete Rodríguez, M.G. (2009). Social support, burnout syndrome and occupational exhaustion among Mexican traffic police agents. The Spanish Journal of Psychology, 12(2), 585-592. DOI: https://doi.org/10.1017/S1138741600001955

Aranda Beltrán, C., Rendon Lizárraga, R. E. \& Ramos Guitérrez, J. A. (2011). Síndrome de burnout y salud en trabajadores del sistema de tren eléctrico. En Revista Waxapa, 3(5), 89-93.

Arellano Gault, D. \& Coronilla Cruz, R. (2012). La corrupción institucionalizada en México. En Arellano David. (Ed.) ¿Podemos reducir la corrupción en México? Límites y posibilidades de los instrumentos a nuestro alcan$c e$. México: Centro de Investigación y Docencia Económicas.

Arnold D., \& Spedding, A. (2005). Mujeres en los movimientos sociales en Bolivia. 2000-2003. (No. CD-IICA-: E50. M9-A7m). La Paz: ILCA-CIEM.

Auné, S. E., Abal, F. E. \& Attorresi, H. F. (2015). Diseño y construcción de una escala de conducta prosocial para adultos. Revista Iberoamericana de Diagnóstico y Evaluación Psicológica, 42(2), 15-25. DOI: 10.21865/RIDEP42_15

Bagtasos, M. R. (2011). Quality of Work Life: A Review of Literature. DLSU Business \& Economics Review, 20(2), 1-8. https://doi.org/10.3860/ber.v20i2.1909

Balseiro, A. L. (2010). Síndrome de Burnout: factor de riesgo en el personal de enfermería. México: Trillas.

Bazerman, M., Loewenstein, G. \& Moore, D.A. (2002). Why good accountants do bad audits. Harvard Business Review, 11 (80), 96-103.

Boydak Mukadder, O. (2009). A study on primary school teacher burnout levels: the Northern Cyprus case. Education, 129(4), 692-703.

Burke, R. J. \& Greenglass, E. R. (1995). A longitudinal examination of the Cherniss model of psychological burnout. Social Science \& Medicine, 40(10), 1357-1363. https://doi.org/10.1016/0277-9536(94)00267-w

Burke, R. J. (1987). Burnout in police work: An examination of the Cherniss model. Group \& Organization Management, 12(2), 174-188. DOI: https://doi.org/10.1177/105960118701200101

Buzzetti-Bravo, M. (2005). Validación del Maslach Burnout Inventory (MBI). En dirigentes del colegio de profesores A.G. de Chile. Universidad de Chile, Santiago de Chile. Disponible en: http://www.tesis.uchile.cl/tesis/ uchile/2005/buzzetti_m/sources/buzzetti_m.pdf Consultado: 03/03/2016.

Cabrera Pivaral, C. E., Ruiz Cruz, L. K., González Pérez G. J., Vega Lóez, M. G., \& Valadez Figueroa, I. (2009). Efecto de una intervención psicoeducativa para disminuir el Síndrome Burnout en personal de confianza de la Comisión Federal de Electricidad. Salud mental, 32(3) 215-221. https://doi.org/10.17711/SM.01853325.2017.011

Campos Izquierdo, A., Jiménez Beatty, J.E., Martín Rodríguez, M. \& Martínez del Castillo, J. (2007). Calidad de vida y estrés laboral: la incidencia del Burnout en el deporte de alto rendimiento madrileño. Revista Internacional de Ciencias del Deporte, 3(6), 62-77. doi:10.5232/ricyde2007.00605

Caro Hernández, M. (2006). Reseña de: El síndrome de quemarse por el trabajo (burnout). Una enfermedad laboral en la sociedad del bienestar, de Gil-Monte, P. R. Revista de Psicología del Trabajo y de las Organizaciones, 22(1), 123-125.

Cox, T., Kuk, G. \& Leiter, M. (1993). Burnout, Health, Work Stress, and organizational healthiness, En W. B. Schaufeli, C. Maslach, \& T. Marek (Eds.), Professional burnout: Recent developments in theory and research. Series in Applied Psychology: Social Issues and Questions (pp. 177-193). Philadelphia: Taylor \& Francis.

De la Garza Toledo, E. (2013). Trabajo no clásico y flexibilidad. Salvador, 26(68), 315-330. https://doi.org/10.1590/ s0103-49792013000200007

De la Garza Toledo, E. \& Campillo, M. (1998). ¿Hacia dónde va el trabajo humano?, Nueva Sociedad, Septiem- 
bre-Octubre (157), 34-53, Disponible en: http://sgpwe.izt.uam.mx/pages/egt/publicaciones/articulos/hacia_ donde.pdf Consultado: 10/09/2016.

DeFond, M. L. \& Francis, J. R. (2005). Audit research after sarbanes-oxley. Auditing: A Journal of Practice and Theory, 24, (s-1), 5-30. https://doi.org/10.2308/aud.2005.24.s-1.5

Farber, B. (1983). Stress and burnout in the Human Service Professions. New York: Pergamon Press.

Fernández Arata, M. \& Merino Soto, C. (2014). Error de medición alrededor de los puntos de corte en el MBI-GS. Liberabit, 20(2), 209-218.

Figueroa Neri, A. \& Palacios Prieto, A. (2015). Auditorías superiores locales en México: análisis de su normatividad, prácticas y transparencia. Universidad de Guadalajara, Centro Universitario de Ciencias Económico Administrativas.

Fogarty, T. J., Singh, J., Rhoads, G. K., \& Moore, R. K. (2000). Antecedents and consequences of burnout in accounting: Beyond the role stress model. Behavioral Research in Accounting, 12, 31. DOI: 10.1111/acfi.12049

Gil-Monte, P. R. (2006). El síndrome de quemarse por el trabajo (burnout). Factores antecedentes y consecuentes. En P. R. Gil-Monte, M. Salanova, J. L. Aragón y W. Schaufeli (Compiladores), Jornada "El Síndrome de quemarse por el trabajo en Servicios Sociales” (pp. 11-25). Valencia: Diputación de Valencia.

Gil-Monte, P. R. (2005). El síndrome de quemarse por el trabajo, (burnout).Una enfermedad laboral en la sociedad del bienestar. Madrid: Pirámide. DOI: 10.13140/2.1.4614.8806

Gil-Monte, P., Núñez-Román, E. \& Selva-Santoyo, Y. (2006). Relación entre el Síndrome de Quemarse por el Trabajo (Burnout) y síntomas cardiovasculares: un estudio en técnicos de prevención de riesgos laborales. Interamerican Journal of Psychology, 40, (2), 227-232.

Gil-Monte, P. R. (2003). Burnout syndrome: ¿síndrome de quemarse por el trabajo, desgaste profesional, estrés laboral o enfermedad de Tomás?, Revista de Psicología del Trabajo y de las Organizaciones, 19(2), 181-197. Disponible en: http://www.redalyc.org/articulo.oa?id=231318052004 Consultado: 7/08/2017.

Gil-Monte, P.R. (2002a). Influencia del género sobre el proceso de desarrollo del síndrome de quemarse por el trabajo (burnout) en profesionales de enfermería. Psicologia em Estudo, 7(1) 3-10. http://dx.doi.org/10.1590/ S1413-73722002000100003

Gil-Monte, P. R. (2002b). Validez factorial de la adaptación al español del Maslach Burnout Inventory-General Survey. Revista Salud Pública de México, 44(1) 33-40. Disponible en: http://www.insp.mx/salud/index.html Consultado: 7/08/2017.

Gil-Monte, P. \& Peiró, J. M. (1999).Validez factorial del Maslach Burnout Inventory en una muestra multiocupacional, Psicothema, 11(3), 679-689.

Gil-Monte, P. \& Peiró, J.M. (1997). Desgaste psíquico en el trabajo: el síndrome de quemarse. Madrid: Síntesis.

Golembiewski, R., Munzenrider, R. \& Carter, D. (1983). Phases of progressive burnout and their work site covariants: Critical issues in OD research and praxis. The Journal of applied behavioral science, 19(4), 461-481. https://doi.org/10.1177/002188638301900408

Guerrero Barona, E. \& Rubio Jiménez, J. C. (2005). Estrategias de prevención e intervención del Burnout en el ámbito educativo, Revista Salud Mental, 28(5), 27-33. https://doi.org/10.17711/SM.0185-3325.2017.011

Guillén Villegas, J. C. \& Santamaría Barberán, E, (2012). Evaluación del nivel de Burnout en una muestra de trabajadores del área de tratamiento de un Centro Penitenciario. Revista Española de Sanidad Penitenciaria, 1(3), 68- 72.

Guthrie, C.P., \& Jones III, A. (2012). Job burnout in public accounting: understanding gender differences. Journal of Managerial Issues, 24(4), 390-411.

Hair, J., Black, W., Babin, B., Anderson, R. \& Tatham, R. (1998). Multivariate data analysis. Estados Unidos: Pearson.

Halbesleben, J. R., \& Demerouti, E. (2005). The construct validity of an alternative measure of burnout: Investigating the English translation of the Oldenburg Burnout Inventory. Work \& Stress, 19(3), 208-220.

Hobfoll, S. \& Freedy, J. (1993). Conservation of resources: A general stress theory applied to burnout. In Wilmar, Schaufeli, Christina, Maslach and Tadeusz, Marek (Eds.), Professional burnout: Recent developments in theory and research. London: Taylor and Francis. 
Jones III, A., Guthrie, C.P., \& Iyer, V.M. (2012). Role Stress and Job Outcomes in Public Accounting: Have the Gender Experiences Converged?. In Advances in Accounting Behavioral Research (pp. 53-84). Emerald Group Publishing Limited. DOI: 10.1108/S1475-1488(2012)0000015007

Juárez-García, A., Idrovo, A., Camacho-Ávila, A. \& Placencia-Reyes, O. (2014). Síndrome de burnout en población mexicana: Una revisión sistemática. Revista Salud mental, 37(2), 159-176. https://doi.org/10.17711/ SM.0185-3325.2017.011

Khan, M. (2006). Role of audit in fighting corruption. Nueva York: Organización de las Naciones Unidas.

Kristensen, T. S., Borritz, M., Villadsen, E., \& Christensen, K. B. (2005). The Copenhagen Burnout Inventory: A new tool for the assessment of burnout. Work \& Stress, 19(3), 192-207. DOI: 10.1080/02678370500297720

Lámbarry Vilchis, F., Rivas Tovar, L. A. \& Trujillo Flores, M. (2013). Desarrollo de una escala de medición de la percepción en la calidad del servicio en los sistemas de autobuses de tránsito rápido a partir del metrobús de la Ciudad de México. Revista Innovar, 23(50), 79-92. Disponible en: http://www.redalyc.org/articulo. oa? id=81828692007 Consultado: 3/03/2017.

Landero, R. \& González, M. (2009). Estadística con SPSS y metodología de la investigación. México: Trillas.

Lee, R. T. \& Ashforth, B. E. (1993). A longitudinal study of burnout among supervisors and managers: Comparisons between the Leiter and Maslach (1988) and Golembiewski et al., (1986) models. Organizational Behavior and Human Decision Processes, 54(3), 369-398. https://doi.org/10.1006/obhd.1993.1016

Leiter, M. \& Maslach, C. (1988). The impact of interpersonal environment on burnout and organizational commitment. Journal of organizational behavior, 9(4), 297-308. 10.1002/job.4030090402.

Leiter, M. P., Clark, D . \& Durup, J. (1994). Distinct models of burnout and commitments among men and women in the military. Journal of applied behavioral science, 30(1), 63-82. https://doi.org/10.1177/0021886394301004

Loaiza, B., \& Peña, A. E. (2013). Niveles de estrés y síndrome de Burnout en contadores públicos colombianos. Actualidad contable FACES, 16(26), 27-44. Disponible en: http://www.redalyc.org/articulo.oa?id=25728399003 Consultado: 17/05/2016.

Malach-Pines, A. (2005). The burnout measure, short version. International Journal of Stress Management, 12(1), 78-88. doi:10.1037/1072-5245.12.1.78

Martínez Martínez, I. M. \& Salanova Soria, M. (2003). Niveles de burnout y engagement en estudiantes universitarios. Relación con el desempeño y desarrollo profesional. Revista de Educación, 330, 361-384.

Maslach, C \& Jackson, S. (1985). The role of sex and family variables in burnout. Sex roles, 12(7/8), 837-851. DOI: 10.1007/BF00287876

Maslach, C., Jackson, S. \& Leiter, M. (1996). Maslach burnout inventory. Third Edition. Palo Alto, CA: Consulting Psychologist Press.

Maslach, C. \& Schaufeli, W. (1993). Historical and conceptual development of burnout. in Schaufeli, Wilmar, Maslach, Christina and Marek, Tadeusz. (Eds), Professional Burnout: Recent Developments in Theory and Research, Washington, DC: Taylor and Francis.

McCarty, W. P., Solomon-Zhao, J. \& Garland, B. E. (2007). Occupational stress and burnout between male and female police officers: Are there any gender differences?, Policing: An International Journal of Police Strategies and Management, 30(4), 672-691. doi:10.1108/13639510710833938

Moore, D. A., Tetlock, P. E., Tanlu, L. \& Bazerman, M. (2006). Conflicts of interest and the case of auditor independence: Moral seduction and strategic issue cycling. Academy of Management Review, 31(1), 10-29. https:// doi.org/10.5465/amr.2006.19379621

Moreno, B. \& Peñacoba, C. (1999). El estrés asistencial en los servicios de salud. En M. A. Simón (Eds). Psicología de la salud. Madrid, España: Biblioteca Nueva.

Moreno Jiménez, J.C., Trujillo Flores, M., Rivas Tovar, L.A. \& Lámbarry Vilchis, F. (2014). Evolution of the Concept and Models of Work Exhaustion (Burnout): The Research in Mexico. International Business Research, 7(9), 45-66. http://dx.doi.org/10.5539/ibr.v7n9p45

Moreno-Jiménez, B., Rodríguez-Carvajal, R. y Escobar, E. (2001). La evaluación del burnout profesional. Factorialización del MBI-GS. Un análisis preliminar. Ansiedad y estrés, 7(1), 69-78. 
Moreno-Jiménez, P. M., Ríos-Rodríguez, M. L, Canto-Ortíz, J., San Martín-García, J. y Perles-Nova, F. (2010). Satisfacción laboral y Burnout en trabajos poco cualificados: diferencias entre sexos en población inmigrante. Revista de Psicología del Trabajo y de las Organizaciones, 26(3), 255-265. DOI: 10.5093/tr2010v26n3a8

Moriana Elvira, J. \& Herruzo Cabrera, J. (2004). Estrés y burnout en profesores. International journal of clinical and health psychology, 4(3), 597-621.

Munro, E. (2004). The impact of audit on social work practice. The British Journal of Social Work, 34(8), 10751095. https://doi.org/10.1093/bjsw/bch130

Olivares Faúndez, V. E. \& Gil Monte, P. R. (2009). Análisis de las principales fortalezas y debilidades del "Maslach Burnout Inventory" (MBI). Ciencia \& Trabajo, 11(33), 160-167.

Oramas, A., González, A. \& Vergara, A. (2007). El desgaste profesional. Evaluación y factorialización del MBI-GS. Revista Cubana de Salud y Trabajo, 8(1), 37-45.

Ordenes, N. (2004). Prevalencia de Burnout en trabajadores del hospital Roberto del Río. Revista Chilena de Pediatría, 75(5), 449-454 http://dx.doi.org/10.4067/S0370-41062004000500006

Pardinas, J. (2009). Instituciones para la Rendición de Cuentas en México. Ed. Ackerman, J.M. La autonomía constitucional de la Auditoría Superior de la Federación (No. 524). Universidad Nacional Autónoma de México, Instituto de Investigaciones Jurídicas.

Peinado Portero, A. \& Garcés de los Fayos Ruiz, E. J. (1998). Burnout en cuidadores principales de pacientes con Alzheimer: el síndrome del asistente desasistido. Anales de Psicología, 14(1), 83-93.

Power, M. (1997). The audit society. doi:10.1093/acprof:oso/9780198296034.001.0001

Prather-Kinsey, J. (2006). Developing countries converging with developed-country accounting standards: Evidence from South Africa and Mexico. The international journal of accounting, 41(2), 141-162. https://doi. org/10.1016/j.intacc.2006.04.007

Preciado Serrano, M. de L., Salas Sánchez, E. G., Franco Chávez, S. A. \& Vázquez Goño, J. M. (2010). Riesgos psicosociales, burnout y agotamiento emocional laboral en médicos de una institución de beneficencia. Revista Cubana de Salud y Trabajo, 11(3), 3-8.

Price, D. M. \& Murphy, P.A. (1984). Staff burnout in the perspective of grief theory. Death Education, 8(1), 47-58. doi: 10.1080/07481188408251381

Purvanova, R.K. \& Muros, J.P. (2010). Gender differences in burnout: A meta-analysis. Journal of Vocational Behavior, 77(2), 168-185. doi: 10.1016/j.jvb.2010.04.006

Quiceno Japcy. M, \& Vinaccia Alpi, S. (2007). Burnout: "síndrome de quemarse en el trabajo (SQT)". Acta Colombiana de Psicología, 10(2), 117-125.

Reygadas, L. (2004). Las redes de la desigualdad: un enfoque multidimensional. Política y Cultura, (22), 7-25. Disponible en: http://www.redalyc.org/articulo.oa?id=26702202 Consultado: 21/10/2017

Salanova, M., Llorens, S. \& García-Renedo, M. (2003). ¿Por qué se están quemando los profesores?, Revista Prevención, trabajo y salud, 28, 16-20.

Salessi, S. \& Omar, A. (2016). Desarrollo y Validación de una Escala para Medir Actuación Emocional en el Trabajo. Revista Iberoamericana de Diagnóstico y Evaluación Psicológica, (1)41, 66-79. Disponible en: http://www. redalyc.org/articulo.oa?id=459646901007 Consultado: $27 / 11 / 2016$.

Sánchez Díaz, S. G. (2012). "Hacia un concepto ampliado de trabajo. Del concepto clásico al no clásico" de Enrique de la Garza. Nueva Antropología, XXV(76), 137-140. Disponible en: http://www.redalyc.org/articulo. oa?id=15924294008 Consultado: 27/11/2016.

Schaufeli, W. B., Leiter, M. P., \& Maslach, C. (2009). Burnout: 35 years of research and practice. Career development international, 14(3), 204-220. doi 10.1108/13620430910966406

Schwartzmann, L. (2004). Estrés laboral, Síndrome de desgaste (Quemado), Depresión: ¿Estamos hablando de lo mismo? Ciencia \& Trabajo, 6(14), 174-184.

Sefert, K., Jayaratne, S., \& Chess, W. A. (1991). Job satisfaction, burnout, and turnover in health care social workers. Health \& Social Work, 16(3) 193-202. doi:10.1093/hsw/16.3.193

Shirom, A. (2009). Acerca de la validez del constructo, predictores y consecuencias del burnout en el lugar de trabajo. Ciencia y Trabajo, 11(32), 44-54. 
Shirom, A. (2003). Job-related burnout. En J. C. Quick y L. E. Tetrick (Eds). Handbook of occupational health psychology (pp. 245-265). Washington, DC: American Psychological Association.

Soto Cámara, R., \& Inmaculada Santamaría Cuesta, M. (2005). Prevalencia del burnout en la enfermería de atención primaria. Enfermería clínica, 15(3), 123-130. https://doi.org/10.1016/s1130-8621(05)71098-6

Tomás Da Silva, T., Lima Oliveira, A. \& Pacheco Miguel, J. (2015). Adaptacáo e validacáo transcultural de uma medida de atitudes acerca da estatística. Revista Iberoamericana de Diagnóstico y Evaluación Psicológica, 1(39), 102-112. Disponible en: http://www.redalyc.org/articulo.oa?id=459645431010 Consultado: 13/12/2016.

Ugalde, L. (2002). La rendición de cuentas en los gobiernos estatales y municipales. Cámara de Diputados, LVIII Legislatura, Comisión de Vigilancia, México.

Valdez, H. (2009). Detección y tratamiento del Síndrome de Burnout en el sistema penitenciario de Jalisco. Revista Waxapa, 1 (1), 24-34.

Volpp, K. G. \& Grande, D. (2003). Resident's suggestions for reducing errors in teaching hospitals. New England Journal of Medicine, 348(9) 851. doi: 10.1056/NEJMsb021667

Winnubst, J. (1993). Organizational structure, social support, and burnout. In Wilmar Schaufeli, Christina, Maslach and Tadeusz, Marek (Eds.), Professional burnout: Recent developments in theory and research, London: Taylor and Francis.

Zaldúa, G., Lodiue, M. \& Koloditzky, D. (2000). La salud de los trabajadores de la salud. Estudio del síndrome de Burnout en hospitales públicos de la ciudad autónoma de Buenos Aires. Revista del Hospital Materno Infantil Ramón Sarda, 19(4), 167-172.

\section{Anexos}

Tabla A1

Muestreo basado en cuotas del ámbito federal

\begin{tabular}{ccccc}
\hline Ejecutivo & Legislativo & Judicial & Mujeres & Hombres \\
\hline 53 & 36 & 0 & 62 & 27 \\
\hline
\end{tabular}

Fuente: elaboración propia. 
Tabla A2

Muestreo basado en cuotas: Entidad de Fiscalización Superior Local EFSL y Contraloría Interna de los Estados CIE.

\begin{tabular}{|c|c|c|c|c|}
\hline Entidad & EFSL & CIE & Mujeres & Hombres \\
\hline Aguascalientes & 4 & 0 & 1 & 3 \\
\hline Baja California & 9 & 6 & 7 & 8 \\
\hline Baja California Sur & 3 & 0 & 0 & 3 \\
\hline Campeche & 2 & 5 & 3 & 4 \\
\hline Chiapas & 7 & 8 & 6 & 9 \\
\hline Chihuahua & 15 & 0 & 12 & 3 \\
\hline Ciudad de México & 12 & 11 & 7 & 16 \\
\hline Coahuila & 6 & 3 & 3 & 6 \\
\hline Colima & 5 & 4 & 8 & 1 \\
\hline Durango & 0 & 7 & 5 & 2 \\
\hline Estado de México & 14 & 8 & 9 & 13 \\
\hline Guanajuato & 0 & 17 & 5 & 12 \\
\hline Guerrero & 6 & 5 & 4 & 7 \\
\hline Hidalgo & 9 & 4 & 6 & 7 \\
\hline Jalisco & 5 & 6 & 8 & 3 \\
\hline Michoacán & 8 & 6 & 4 & 10 \\
\hline Morelos & 7 & 2 & 3 & 6 \\
\hline Nayarit & 4 & 11 & 6 & 9 \\
\hline Nuevo León & 5 & 13 & 6 & 12 \\
\hline Oaxaca & 9 & 8 & 4 & 13 \\
\hline Puebla & 0 & 7 & 0 & 7 \\
\hline Querétaro & 11 & 5 & 10 & 6 \\
\hline Quintana Roo & 17 & 3 & 5 & 15 \\
\hline San Luis Potosí & 7 & 5 & 2 & 9 \\
\hline Sinaloa & 9 & 4 & 6 & 7 \\
\hline Sonora & 15 & 0 & 3 & 12 \\
\hline Tabasco & 11 & 0 & 4 & 8 \\
\hline Tamaulipas & 3 & 5 & 2 & 6 \\
\hline Tlaxcala & 7 & 11 & 2 & 16 \\
\hline Veracruz & 13 & 12 & 8 & 17 \\
\hline
\end{tabular}

Nota: Totales ESFL 239. CIE: 187. Mujeres: 158. Hombres: 268.

Fuente: elaboración propia. 